\title{
Comparison of Death Rate in Hospitalized and Non Hospitalized Intensive Care Patients of Masih Daneshvari Hospital
}

\author{
Seyed Sajjad Razavi, ${ }^{1}$ Hamidreza Jamaati, ${ }^{2}$ Mehrzad Ghasemzadeh, ${ }^{3}$ and Sara Salarian ${ }^{4,}$ \\ ${ }^{1}$ Anesthesiology Research Center, Mofid Children Hospital, Shahid Beheshti University of Medical Sciences, Tehran, IR Iran \\ ${ }^{2}$ Chronic Respiratory Diseases, National Research Institute of Tuberculosis and Lung Diseases, Shahid Beheshti University of Medical Sciences, Tehran, IR Iran \\ ${ }^{3}$ Department of Critical Care Medicine, Guilan University of Medical Sciences, Rasht, IR Iran \\ ${ }^{4}$ Department of Critical Care Medicine, Shahid Beheshti University of Medical Sciences, Tehran, IR Iran \\ "Corresponding author: Sara Salarian, Department of Critical Care Medicine, Mofid Children Hospital, Shahid Beheshti University of Medical Sciences, Tehran, IR Iran. Tel: \\ +98-2122259004, Fax: +98-2189783447, E-mail: sarasalarian@yahoo.com
}

Received 2015 September 30; Accepted 2015 November 14.

\begin{abstract}
Objectives: The aim of this study was to compare death rate in accepted patients in Masih Daneshvari hospital intensive care from 30th of November 2011 to 30th of December 2011 in comparison with unaccepted patients to the intensive care department.

Methods: The research was done at Masih Daneshvari hospital on patients that had referred to the emergency, and hospitalized patients at the intensive care department. Overall, 170 candidates, who were hospitalized at intensive care were entered in the study with the method of aimed-incidental samplings. The information-collecting tool was a three-part survey; the first part included demographic information, the second part investigated acceptance and release factors in intensive care, and the third was the acute physiology and chronic health evaluation (APACHE II) tool. The collected data were analysed using the SPSS software.

Results: Amongst the 170 candidates hospitalised at the intensive care unit (ICU), 81 people could enter the ICU after waiting three days, and the 89 remaining couldn't enter the ICU. The death rate in hospitalized candidates in ICU was 32.09 and for those who couldn't make it (not having entered the ICU) was 32.58. The average APACHE mark in ICU hospitalized patients was $12.00 \pm 6.63$ and for those who couldn't be hospitalized was $11.12 \pm 6.69$.

Conclusions: On the basis of this study results, the APACHE score average and the death rate of those not able to enter the ICU in comparison with the hospitalized cases didn't have a significant difference ( $P \geq 0.05)$. The common cause of patients death at intensive care was pneumonia, while deaths were more common amongst males and the elderly.
\end{abstract}

Keywords: Death Rate, Intensive Care Department, APACHE Average Score

\section{Background}

The most secure place for severe illnesses is the intensive care unit (1). The intensive care is a specialized section of the hospital where vast, exact and continuous care is provided for the most severe sicknesses (2), and has the most developed technologic medical facilities and the most professional staff. Keeping the patient alive and providing the most qualified care with calmness and patient comfort is one of the most important objectives of intensive care (3). This care is very important for people in critical conditions, who are clinically unstable and potentially threatened by a disorder or severe disease (4). A third of hospital deaths occur in intensive care (2). What happens to patients hospitalized in intensive care is effective on hospital survival and the usage of sources (5). Several factors are influential at the ICU and being aware of them can help the intensive care staff find risk factors and plan to overcome such risks the best way possible and lessen the death rate (4). The quality of care at the ICU can affect death rate of hospitalized patients (6). Due to the limited space of intensive care units, it is necessary to prioritize patients with severe conditions so that they receive greater attention from nurses and doctors in a defined system (7). Systems predicting the situation of very sick patients at intensive care have been developed in developed countries all over the world (5). The recovery rate evaluation and the expected health status on the basis of current methods are generally built on doctor's perception and approximate estimation. Rating of the performance of ICUs is done via analysis and comparison of different centers, considering variables such as treatments effectiveness and allocation of human and financial resources (8). Acute physiology and chronic health evaluation (APACHE II) is one of the most popular general systems. Recently this system of rating has been used in many intensive care centers around the world and hospitals are profiting from its beneficial results. From the 1980 s, most printed studies presented 
information about vital signs either at the time of release from the ICU or when leaving the hospital. A considerable number of patients after being transferred from the ICU died before leaving the hospital (the post ICU death or hidden death) (9). Therefore, regarding the importance of intensive care department and that up to now there has been no research done on the comparison of the number of patient deaths amongst those hospitalized in intensive care and candidates who couldn't enter the ICU, the current research was conducted at Tehran Masih Daneshvari hospital.

\section{Objectives}

This study was done to recognize factors effective on death rate so that in the future upgrading care can lower the death rate.

\section{Methods}

This was a controlled clinical experimental study. The research sample was referred patients to Masih Daneshvari hospital emergency and intensive care department. Overall,170 patients hospitalized in intensive care were entered in the study by the accidental aimed sampling method. The verification was done with three stages: 1 ) verifying all the patients at the hospital emergency unit to make sure if they need to enter the intensive care. 2) patients needing to be hospitalized in intensive care were hospitalized if there were empty beds or were followed after being released for four weeks. 3) if it was not possible to be hospitalized in intensive care, the rate of mortality and morbidity of patients was recorded. The reception and release of patients in intensive care is based on the regulations of the health ministry. After obtaining consent, patients consciously entered the research. The information collection tool had three parts; part 1: demographic information, part 2: reception and release factors in intensive care, and Part 3: the APACHE index. All the clinical discoveries by intensive care staff were considered as internal plan credit. Collected information was analyzed by the SPSS version 16 software, using the Chi-square, independent T and Mann-Whitney tests.

\section{Results}

The verifications of 170 patients amongst ICU hospitalization candidates indicated that, after an average waiting time of three days, 81 could enter the ICU and the 89 remaining couldn't enter; 45 recovered from their critical situation. Fifteen patients waiting in the emergency unit died, 15 were sent to other hospital departments after diagnosis, and 14 went to other centers. The average APACHE score of hospitalised patients at the ICU was 12/00 $\pm 6 / 63$ and the score of patient who couldn't get hospitalized at the ICU was 11/12 $\pm 6 / 69$. Amongst all the hospitalized patients at the ICU, 26 (32/09) died. The cause of death was pneumonia in eight, tuberculosis in seven, and lung cancer in five. Etymology of hospitalized cases at the ICU in three groups was compared; amongst all the hospitalized candidates at the ICU, 32\% had haemoptysis and $27 \%$ pneumonia, and in non hospitalized patients heart problems and chronic obstructive pulmonary disease (COPD) (24\%) were the most common causes. the most common cause of death of patients at the ICU was pneumonia and in non hospitalised cases was lung cancer. The comparison of death and release rate of patients hospitalised at the ICU (26 deaths and 11 releases) showed that mortality in patients, who couldn't enter the ICU was twice more than the ones hospitalized.

The average hospitalization days at the hospital for ICU patients was 17 days and for those not hospitalized at the ICU was 34 and the difference was considerable $(\mathrm{P}<0.05)$. The death rate comparison of patients not hospitalized at the ICU (32.58), and those hospitalized (32.09), showed that the death rate in the two groups did not have a significant difference $(\mathrm{P}>0.05)$. Also, the average APACHE in the two groups accepted or not accepted at the ICU did not have a significant difference $(\mathrm{P}>0.05)$ (Table 1$)$.

Table 1. The Acute Physiology and Chronic Health Evaluation Score of Hospitalised and Non-Hospitalised Patients at the Intensive Care Unit

\begin{tabular}{lcc}
\hline & \multicolumn{2}{c}{ Frequency and APACHE Mark } \\
\cline { 2 - 3 } Patients & Number & APACHE Mark \\
\hline $\begin{array}{l}\text { All candidates for hospitalization at the } \\
\text { ICU }\end{array}$ & 170 & $11 / 4$ \\
\hline ICU candidates total death number & 55 & $16 / 9$ \\
\hline Hospitalized patients at the ICU & 81 & $11 / 85$ \\
\hline Death at the ICU & 26 & $15 / 9$ \\
\hline Non hospitalized at the ICU & 89 & $10 / 9$ \\
\hline Death out of the ICU & 29 & 17 \\
\hline
\end{tabular}

\section{Discussion}

In this research, which was performed between February 2011 and the beginning of February 2012, from 81 hospitalized patients at the ICU 23 died; $73.07 \%$ were male and 26.92\% were female. The comparison of death rate based on gender in all hospitalized candidates at the ICU showed 
that the death rate in males was higher and the relationship of death rate and gender was considerable $(\mathrm{P}<0.05)$; this is consistent with the study of Golnuk, in which 69.3\% of males and $30.7 \%$ of females died (10). These findings are also compatible with the study of Feizi from Iran that was done temporarily at the intensive care and brain surgery units $(72.8 \%$ of deaths occurred in males while $72.2 \%$ was observed in females) (11). In the study of Ramazani (7), the mortality rate was $58 \%$ in males and $42 \%$ in females, where there was no significant gender difference, therefore it was different from this study's results despite the fact that the study period was six months. In this research regarding the obtained results in one year, the death rate was $32.35 \%$ of all hospitalized patients, while in the study of Golnuk this rate was $15.6 \%$ (10), in the study of Feizi 15.5\% (11) and in the study of Mohammadi 22\% (12). Regarding the discoveries of this study, the most common cause of death at the ICU was pneumonia (27\%). In Feizi study the most common cause of death was brain damage caused by injury (41/4\%) (11) and in Zand's study, heart arrhythmia was the most common cause (28.1\%) (13). Also the average APACHE score of patients that died and those who survived at the ICU didn't have a significant difference $(P>0.05)$. In a retrospective study by Rahimzadeh, it was shown that amongst patients staying alive after an ICU stay, the average APACHE score was 11.75 and in those who died, this score was 22/.6, with the difference being significant (14). This difference may be due to the difference in the sample size of the two studies. In another study, Ramazani reported that in people with high APACHE score, the death rate was higher than those with a low APACHE score. The APACHE score decreased in the first 24 hours of hospitalization and had a significant statistical relationship with patients' death rate (7), which was different from this study's results. The difference may be due to the difference in the durations of the study periods.

The average APACHE score in patients who died and those who survived outside the ICU had a significant statistical difference $(\mathrm{P}<0.05)$, which shows that APACHE is considerable out of the ICU from the pre-awareness point of view. This finding is compatible with the study of Goel, who showed APACHE results were a predictive tool for death in COPD patients' death treated in hospital units other that the ICU (15).

Because Masih Daneshvari hospital is one of the most important treatment centers in Iran, the information derived from this study can be generalized to all the intensive care departments of other hospitals having a similar problem. Regarding the fact that half of the patients couldn't enter the ICU, it is suggested that the number of beds should at least be doubled, and considering that the majority of deaths were caused by pneumonia, it is sug- gested that the intensive care personnel should respect the regulations of disinfection techniques of patients' respiratory airways.

\section{Acknowledgments}

We thank the anastasia group professors, the intensive care personnel, emergency and intensive care colleagues of Masih Daneshvari hospital in Tehran for their cooperation and help with this project.

\section{References}

1. Gupta S, Bhagotra A, Gulati S, Sharma J. Guidelines for the transport of critically Ill patients. JK Sci. 2004;6(2):109-12.

2. Ghoneim AHA, Hussein RM, El-Ghamry R, Mahmoud LY. Patterns of admitted cases to Respiratory Intensive Care Unit at Zagazig University Hospitals, Egypt. Egypt J Chest Dis Tuberc. 2013;62(4):661-8. doi: 10.1016/j.ejcdt.2013.09.003.

3. Ratanarat R, Thanakittiwirun M, Vilaichone W, Thongyoo S, Permpikul C. Prediction of mortality by using the standard scoring systems in a medical intensive care unit in Thailand. J Med Assoc Thai. 2005;88(7):949-55. [PubMed: 16241025].

4. Janmohammadi N, Alijanpour E, Bahrami M, Taheri M, Hosseni H. Outcome of the Patients Admitted to the Surgical Intensive Care Unit of Shahid Beheshti Hospital (Babol, Iran). J Babol Univ Med Sci. 2014;16(6):72-7.

5. Abelha F, Maia P, Landeiro N, Neves A, Barros H. Determinants of outcome in patients admitted to a surgical intensive care unit. Arq Med. 2007;21(5,6):135-43.

6. Sadaka F, Palagiri A, Trottier S, Deibert W, Gudmestad D, Sommer SE, et al. Telemedicine intervention improves ICU outcomes. Crit Care Res Pract. 2013;2013:456389. doi: 10.1155/2013/456389. [PubMed: 23365729].

7. Ramazani J, Hosseini M. The Assessment of APACHE II Scoring System in Predicting the Result of Weaning from Ventilator [in Persian]. J Danesh Tandorosti. 2014;8(4):187-92.

8. Mohammadi H, Haghighi M. The Assessment of mortality in ICU with different APACHE II scores [in Persian]. J Guilan Univ Med Sci. 2006;15(59):85-90.

9. Moreno R, Agthe D. ICU discharge decision-making: are we able to decrease post-ICU mortality?. Intensive Care Med. 1999;25(10):1035-6. [PubMed: 10551955].

10. Goulenok C, Monchi M, Chiche JD, Mira JP, Dhainaut JF, Cariou A. Influence of overweight on ICU mortality: a prospective study. Chest. 2004;125(4):1441-5. [PubMed: 15078757].

11. Feizi I, Ansari M. Mortality and risk factors in patients admitted to the intensive care unit. J Ardabil Univ Med Sci. 2008;8(4):420-3.

12. Mohammadi H, Haghighi M. Survey relationship of mortality rate of hospitalized patients in ICU with different degrees of APACHE II [in Persian]. J Guilan Univ Med Sci. 2006.

13. Zand S, Rafiei M. Causes and duration of hospitalization and mortality rate in intensive care units in Central province. J Crit Care Nurs. 2010;3(2):7-8.

14. Rahimzadeh $P$, Taghipur Anvari Z, Hassani V. Estimation of mortality rate of patients in surgical intensive care unit of Hazrat-Rasul hospital [in Persian]. Hakim Res J. 2008;11(1):22-8.

15. Goel A, Pinckney RG, Littenberg B. APACHE II predicts long-term survival in COPD patients admitted to a general medical ward. J Gen Intern Med. 2003;18(10):824-30. [PubMed:14521645]. 